\title{
Analytical STEM for metal-organic frameworks (MOFs) and MOF composites
}

\section{Sean Collins}

University of Leeds, United States

Analytical signals in scanning transmission electron microscopy (STEM), including diffraction and spectroscopic signals in addition to the angle-selective bright field (BF) and annular dark field imaging (ADF) modalities, have seen diverse application targeting nanoscale structure-chemistry relationships in metals, inorganic semiconductors, ceramics, and two-dimensional materials. These approaches are increasingly viable for hybrid and other molecular materials containing molecular subunits. Molecular materials like metal-organic frameworks (MOFs), hybrid, and organic materials exhibit critical electron doses as low as $<10 \mathrm{e}^{-} \AA^{-2}$ setting substantial challenges for controlling and monitoring beam-sample interactions for access to information on the native state of molecular solids. This presentation will discuss advances for diffraction, spectroscopy, and imaging for accessing multiscale and correlative information for MOF and MOF composite materials.

MOFs, comprised of metal cations or metal clusters connected by organic linker molecules in an extended network, exhibit properties of substantial interest for applications in gas storage, separations, and catalysis. The development of MOF glasses and their blends and composites has introduced shapable, monolithic materials with tunable mechanical and chemical properties. The composite microstructures possible in MOF crystals, blends, and crystal-glass composites have prompted efforts to interrogate interfaces and grain structure in MOFs.

Minimization of electron dose, including through the use of electron counting detectors for imaging, have enabled structure solution ${ }^{1}$ and high resolution TEM of MOFs. ${ }^{2}$ Beyond these important results, hierarchical structure of MOFs and emerging MOF-composite materials require spatially resolved analytical measurements across length scales approaching microns in samples exhibiting feature sizes and concomitant sample thickness in excess of $100 \mathrm{~nm}$ while preserving nanometre-scale detail.

Low-dose scanning electron diffraction (SED) with direct electron detection has proven to access structural information on these scales for the identification of crystalline domains in MOF crystal-glass composites and virtual dark field imaging of correlated defect nano-domains in UiO-66 (Figure 1). Coupled with lattice-resolution imaging in STEM, these analyses provide insight into faceting, domain orientation and size, and surface termination in UiO-66 crystals. This presentation will showcase work on additional defect structures in MOFs beyond defect domains.

Structural insights from spatially resolved diffraction and imaging, however, provides incomplete information on chemically-diverse MOF materials, prompting correlative X-ray energy dispersive spectroscopy mapping to reveal $^{3}$ as well as core-shell structures. ${ }^{4}$ Signatures of metal-coordination geometry ${ }^{5}$ and functional group vibrations ${ }^{6}$ have also been realised using ultrahigh resolution monochromated EELS (SuperSTEM, UK).

In this presentation, recent results on correlative electron tomography and point diffraction and correlative SED and cathodoluminescence (CL) will be discussed, with an emphasis on optoelectronic MOF composite materials with promising control over luminescence characteristics. 


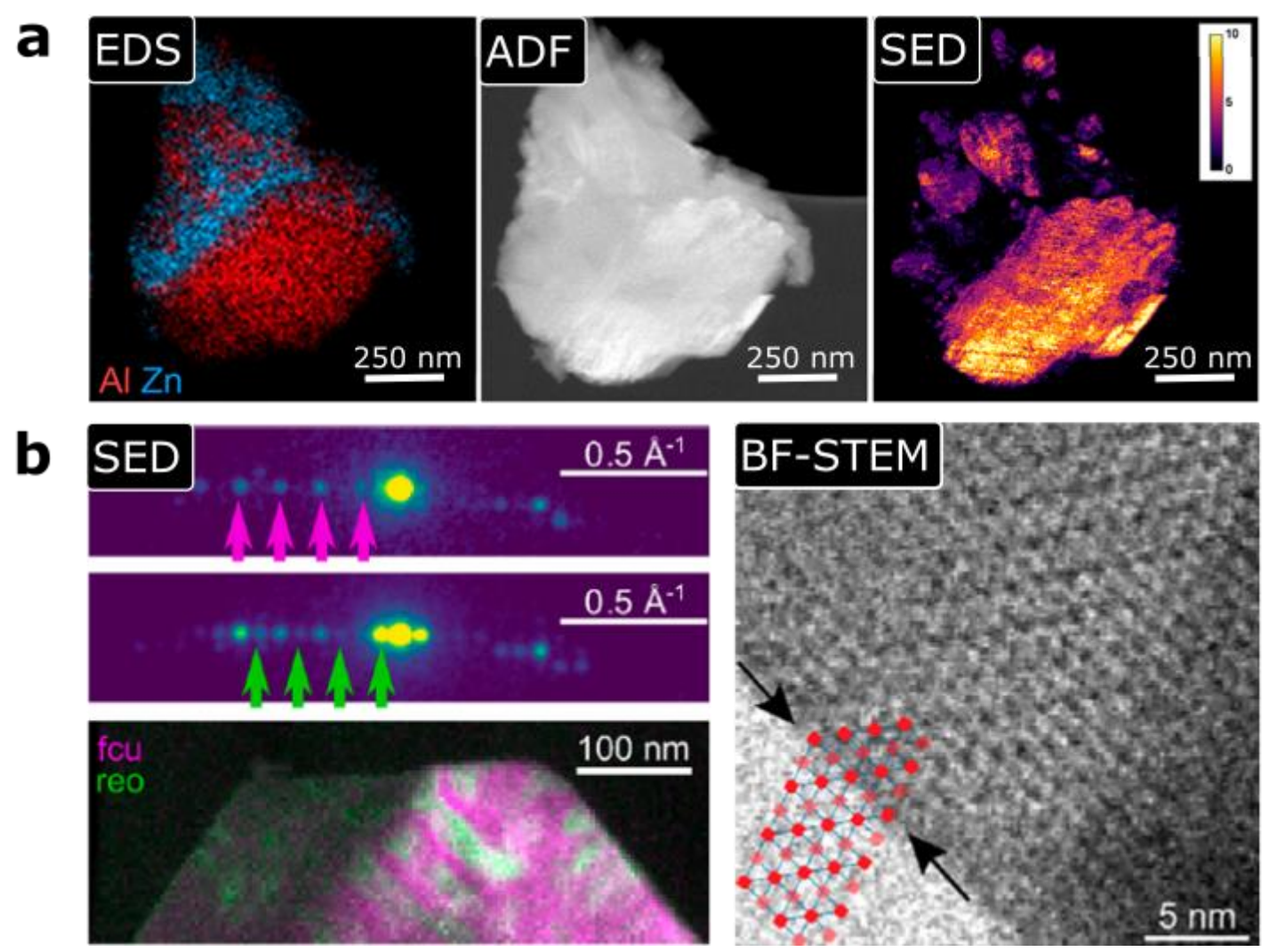

Figure 1. a, Chemical and structural imaging of MIL-53/ZIF-62 crystal-glass composites. Adapted from Ref. 3 (CC-BY). b, Diffraction- and high resolution BF-STEM imaging for characterisation of defect nano-domains in UiO-66 crystals. Adapted from Ref. 7 (CC-BY).

References

(1) Wang, B.; et al. Chem. Eur. J. 2018, 24 (66), 17429-17433.

(2) Liu, L.; et al. Nat. Chem. 2019, 11 (7), 622-628.

(3) Ashling, C. W.; J. Am. Chem. Soc. 2019, 141 (39), 15641-15648.

(4) Orr, K. W. P.; Chem. Sci. 2021. https://doi.org/10.1039/D0SC03940C.

(5) Collins, S. M.; J. Am. Chem. Soc. 2018, 140 (51), 17862-17866.

(6) Collins, S. M.; Nano Lett. 2020, 20 (2), 1272-1279.

(7) Johnstone, D. N.; J. Am. Chem. Soc. 2020, 142 (30), 13081-13089. 